\title{
AN H-INFINITY BASED LOWER BOUND TO SPEED UP THE SPHERE DECODER
}

\author{
M. Stojnic, H. Vikalo, and B. Hassibi \\ California Institute of Technology, Pasadena, CA
}

\begin{abstract}
It is well known that maximum-likelihood (ML) decoding in many digital communication schemes reduces to solving an integer least-squares problem, which is NP hard in the worst-case. On the other hand, it has recently been shown that, over a wide range of dimensions and signal-to-noise ratios (SNR), the sphere decoder can be used to find the exact solution with an expected complexity that is roughly cubic in the dimension of the problem. However, the computational complexity of sphere decoding becomes prohibitive if the SNR is too low and/or if the dimension of the problem is too large. In recent work [7], we have targeted these two regimes and attempted to find faster algorithms by employing a branch-and-bound technique based on convex relaxations of the original integer least-squares problem. In this paper, using ideas from $H^{\infty}$ estimation theory, we propose new lower bounds that are generally tighter than the ones obtained in [7]. Simulation results show the advantages, in terms of computational complexity, of the new $H^{\infty}$-based branch-and-bound algorithm over the ones based on convex relaxation, as well as the original sphere decoder.
\end{abstract}

\section{INTRODUCTION}

In this paper we are interested in solving exactly the following problem

$$
\min _{\mathrm{s} \in \mathcal{D} \subset \mathcal{Z}^{m}}\|\mathbf{x}-H \mathbf{s}\|_{2}
$$

where $\mathrm{x} \in \mathcal{R}^{m}, H \in \mathcal{R}^{m \times m}$, and $\mathcal{D}$ refers to some subset of the integer lattice $\mathcal{Z}^{m}$. The main idea of the sphere decoding algorithm [1] for solving (1) is based on finding all points s such that $H$ s lies within a sphere of some adequately chosen radius $d$ centered at $\mathbf{x}$, i.e., on finding all $\mathbf{s}$ such that

$$
d^{2} \geq\|\mathbf{x}-H \mathbf{s}\|_{2}^{2}
$$

and then choosing the one that minimizes the objective function. Using the $Q R$-decomposition $H=Q R$, with $Q$ unitary and $R$ upper triangular, we can reformulate (2) as

$$
d^{2} \geq\|\mathrm{y}-R \mathrm{~s}\|_{2}^{2}
$$

This work was supported in part by the National Science Foundation under grant no. CCR-0133818, by the Office of Naval Research under grant no. N00014-02-1-0578, and by Caltech's Lee Center for Advanced Networking. where we have defined $\mathbf{y}=Q^{*} \mathbf{x}$.

Now using the upper-triangular property of $R$, (3) can be further rewritten as

$$
\begin{aligned}
d^{2} \geq & \left\|\mathrm{y}_{k: m}-R_{k: m, k: m} \mathrm{~s}_{k: m}\right\|^{2}+ \\
& \left\|\mathrm{y}_{1: k-1}-R_{1: k-1,1: k-1} \mathrm{~s}_{1: k-1}-R_{1: k-1, k: m} \mathrm{~s}_{k: m}\right\|^{2},
\end{aligned}
$$

for any $2 \leq k \leq m$, where the subscripts determine the entries the various vectors and matrices run over. A necessary condition for (3) can therefore be obtained by omitting the second term on the RHS of the above expression to yield

$$
d^{2} \geq\left\|\mathbf{y}_{k: m}-R_{k: m, k: m} \varsigma_{k: m}\right\|^{2} .
$$

The sphere decoder finds all points $s$ in (2) by proceeding inductively on (5), starting from $k=m$ and proceeding to $k=1$. (For more details on the sphere decoder and for an explicit description of the algorithm the reader may refer to $[1,6,3]$.) Although the sphere decoding algorithm introduces the nice idea of searching over the dimensions of the unknown vector one at a time, its worst case complexity is exponential. However, in communications application where the systern model can be described as

$$
\mathrm{x}=H \mathrm{~s}+\mathrm{w},
$$

and where the entries of $w$ represent noise and are independent $\mathcal{N}\left(0, \sigma^{2}\right)$ random variables, it can be shown (see [3]) that for appropriate choice of $d$ the expected complexity of the sphere decoding algorithm is roughly cubic over a wide range of SNR's and dimensions. Still, in low SNR regimes and for large dimensions the complexity of the sphere decoder defined in (5) becomes increasingly prohibitive. In those cases one usually turns to heuristics approaches (see, e.g., [4]) or some kind of statistical pruning (see, e.g. [5]).

In previous work [7], we attempted to reduce the computational complexity of the sphere decoder while still finding the exact solution. The main idea was that instead of using the sphere decoder as defined in (5) we used the one described by

$$
d^{2}-L B \geq\left\|\mathrm{y}_{k: m}-R_{k: m, k: m} \mathrm{~s}_{k: m}\right\|^{2} .
$$

where LB is a lower bound on the RHS of (4), i.e.

$$
\begin{aligned}
& L B=L B\left(\mathbf{y}_{I: k-1}, R_{1: k-1,1: m}, \mathbf{s}_{k: m}\right) \leq \\
& \min _{\mathbf{s}_{: k k-1} \in \mathcal{D} \subset \mathcal{Z}^{k-1}}\left\|\mathbf{y}_{1: k-1}-R_{1: k-1,1: k-1} \mathbf{s}_{1: k-1}-R_{1: k-1, k: m} \mathbf{s}_{k: m}\right\|^{2},
\end{aligned}
$$


As it was noticed in [7], (7) is certainly a more restricted condition than (5) and so will lead to the elimination of more points from the tree. Furthermore, using (7) instead of (5) in the sphere decoder algorithm will not result in missing any lattice points defined by (2) since we use a lower bound for the remainder of the cost in (4).

Clearly, the tighter the lower bound LB, the more points that will be pruned from the tree. This essentially means that in order to obtain a sphere decoder with lower complexity we have to find as tight as possible lower bounds on the integer least-squares problem

$$
\min _{\mathbf{s}_{1: k-1} \in \mathcal{D} \subset Z^{k-1}}\left\|\mathbf{z}_{1: k-1}-R_{1: k-1,1: k-1} s_{1: k-1}\right\|^{2},
$$

where we have defined $\mathbf{z}_{1: k-1}=\mathbf{y}_{1: k-1}-R_{1: k-1, k: \mathrm{m}} \mathbf{s}_{k: m}$. Finding such lower bounds was the focus of [7]. In particular, in [7] we considered two techniques for finding lower bounds on the the integer least-squares problem (8). The first one is based on the relaxation of the discrete search space to a sphere or to a polytope. The second one is based on duality theory. As shown in [7], the duality theory based lower bounds are tighter and therefore will prune the search tree the most. However, they require cubic computational complexity per node visited in the search tree, whereas the spherical relaxation lower bound, say, requires only quadratic complexity. A main conclusion of [7] was that it would be useful to obtain lower bounds tighter than the sphere constrained ones that still require only quadratic complexity per visited node.

In this paper, we address this issue by constructing lower bounds for the integer least-squares problem (8) based on ideas from $H^{\infty}$ estimation theory. We show that computing these bounds requires only quadratic complexity, and that they are tighter than the sphere and polytope constrained bounds of [7], by virtue of the fact that they subsume them as special cases.

For simplicity, in this paper we assume that the symbols are points from a BPSK (or QPSK, in the complex case) constellation. This implies that $\mathcal{D}=\left\{-\frac{1}{2}, \frac{1}{2}\right\}^{k-1}$, However, extensions of the results to higher constellation is straightforward.

\section{H-INFINITY BASED LOWER BOUND}

In this section, we propose finding the lower bound $L B$ in (7) based on $H^{\infty}$ theory. To simplify notation, we rewrite (8) as

$$
\min _{\mathbf{a} \in \mathcal{D} \subset Z^{k-1}}\|\mathbf{b}-L \mathbf{a}\|^{2}
$$

where $\mathbf{a}=\mathbf{s}_{1: k-1}, \mathbf{b}=z_{1: k-1}$, and $L=R_{1: k-1,1: k-1}$.

Consider an estimation problem where $\mathbf{a}$ and $\mathbf{b}-L \mathbf{a}$ are unknown vectors, $b$ is the observation, and the quantities we want to estimate are $\mathbf{a}$ and $\mathbf{b}$. In the $H^{\infty}$ framework, the goal is to construct estimators $\hat{\mathbf{a}}=f_{1}(\mathbf{b})$ and $\hat{\mathbf{b}}=f_{2}(\mathbf{b})$, such that for some given $\gamma$ and $\alpha>0$, we have

$$
\frac{\|\mathbf{a}-\hat{\mathbf{a}}\|^{2}+\|\mathbf{b}-\hat{\mathbf{b}}\|^{2}}{\frac{1}{\alpha}\|\mathbf{a}\|^{2}+\|\mathbf{b}-L \mathbf{a}\|^{2}} \leq \gamma^{2}
$$

for all $\mathrm{a}$ and $\mathrm{b}$ (see, e.g., [8]).

Obtaining the desired lower bound from (10) is now straightforward. Note that for all $a$ and $b$ we can now write

$$
\|\mathbf{b}-L \mathbf{a}\|^{2} \geq \gamma^{-2}\left(\|\mathbf{a}-\hat{\mathbf{a}}\|^{2}+\|\mathbf{b}-\hat{\mathbf{b}}\|^{2}\right)-\frac{1}{\alpha}\|\mathbf{a}\|^{2},
$$

and, in particular,

$$
\begin{aligned}
& \min _{\mathbf{a} \in \mathcal{D}}\|\mathbf{b}-L \mathbf{a}\|^{2} \geq \\
& \min _{\mathbf{a} \in \mathcal{D}}\left(\gamma^{-2}\|\mathbf{a}-\mathbf{a}\|^{2}-\frac{1}{\alpha}\|\mathbf{a}\|^{2}\right)+\gamma^{-2}\|\mathbf{b}-\hat{\mathbf{b}}\|^{2} .
\end{aligned}
$$

Note that the minimization on the RHS of (11) is straightforward since it can be done componentwise. Thus, for any $H^{\infty}$ estimators, $\hat{\mathrm{a}}=f_{1}(\mathrm{~b})$ and $\hat{\mathrm{b}}=f_{2}(\mathrm{~b})$, (11) provides a readily computable lower bound. The issue, of course, is how best to obtain $\hat{\mathbf{a}}$ and $\hat{\mathbf{b}}$ (and $\alpha$ and $\gamma$ ). To this end, let us assume that the estimators are linear, i.e., $\hat{\mathbf{a}}=K_{1} \mathrm{~b}$ and $\hat{\mathbf{b}}=$ $K_{2} \mathbf{b}$ for matrices $K_{1}$ and $K_{2}$ of the appropriate size. Introducing $\mathbf{c}=\left[\begin{array}{c}\frac{1}{\sqrt{\alpha}} \mathbf{a} \\ \mathbf{b}-L \mathbf{a}\end{array}\right]$ and $\mathcal{T}=\left[\begin{array}{cc}\sqrt{\alpha}\left(I-K_{1} L\right) & -K_{1} \\ \sqrt{\alpha}\left(I-L K_{2}\right) L & I-L K_{2}\end{array}\right]$ we have from (11) that for all $\mathbf{c}$ it must hold that

$$
\mathbf{c}^{*} T^{*} T \mathbf{c} \leq \gamma^{2} \mathbf{c}^{*} I \mathrm{c},
$$

(see [8]). This implies the matrix inequality

$$
T^{*} T \leq \gamma^{2} I
$$

The tighter the bound in (12), the tighter will be the bound in (11). Hence we will attempt to choose $K_{1}$ and $K_{2}$ to make $\gamma^{-2} T^{*} T$ as close to identity as possible.

After multiplying the left-hand side with the unitary ma$\operatorname{trix}\left[\begin{array}{cc}\nabla^{-1} & \sqrt{\alpha} L^{*} \Delta^{-*} \\ -\sqrt{\alpha} L \nabla^{-1} & \Delta^{-*}\end{array}\right]^{*}$, and the right-hand side with $\left[\begin{array}{cc}\nabla^{-1} & \sqrt{\alpha} L^{*} \Delta^{-*} \\ -\sqrt{\alpha} L \nabla^{-1} & \Delta^{-*}\end{array}\right]$, where $\alpha L^{*} L+I=\nabla^{*} \nabla$ and $\alpha L L^{*}+I=\Delta \Delta^{*}$, and noting that $T=\left[\begin{array}{cc}\sqrt{\alpha} I & 0 \\ \sqrt{\alpha} L & I\end{array}\right]-$ $\left[\begin{array}{c}K_{1} \\ L K_{2}\end{array}\right]\left[\begin{array}{ll}\sqrt{\alpha} L & I\end{array}\right],(12)$ can be reformulated as

$$
\left[\begin{array}{cc}
A A^{*}+B B^{*} & B C^{*} \\
C B^{*} & C C^{*}
\end{array}\right] \leq \gamma^{2} I,
$$

where $A=\sqrt{\alpha} \nabla^{-I}, B=\alpha L^{*} \Delta^{-*}-K_{1} \Delta$, and $C=$ $\left(I-L K_{2}\right) \Delta$. We have many degrees of freedom. Let us set 
$C^{*} C=\gamma_{1}^{2} I$, for some $0<\gamma_{1}<\gamma$. To make half the eigenvalues of $\gamma^{-2} T^{*} T$ unity, we can now set the Schur complement of the $(1,1)$ entry to zero, i.e., $\left(\gamma^{2}-\gamma_{1}^{2}\right) I-C B^{*}\left(\gamma^{2} I-\right.$ $\left.\left(A A^{*}+B B^{*}\right)\right)^{-1} B C^{*}=0$. From these two, it easily follows that $B B^{*}=\left(1-\frac{\gamma_{1}^{2}}{\gamma^{2}}\right)\left(\gamma^{2} I-A A^{*}\right)$. Furthermore, for all this to hold, we must have $\gamma^{2} I-\left(A A^{*}+B B^{*}\right) \geq 0$ or, in other words, $\gamma^{2} \geq \frac{1}{\frac{1}{\alpha}+\lambda_{\min }\left(L^{*} L\right)}$. Putting everything together, we establish a lower bound on the value of the objective function in (9) $L B_{\text {hinf }}$ as

$$
L B_{\text {hinf }}=\min _{\mathbf{a} \in \mathcal{D}} \gamma^{-2}|| \mathbf{a}-K_{1} \mathbf{b}\left\|^{2}+\frac{\gamma_{1}^{2}}{\gamma^{2}}\right\| \Delta^{-1} \mathbf{b} \|^{2}-\frac{k-1}{4 \alpha},
$$

where $K_{1}=\alpha L^{*}\left(\alpha L L^{*}+I\right)^{-1}-B \Delta^{-1}, B$ is any matrix such that $\left.B B^{*}=\left(1-\frac{\gamma_{1}^{2}}{\gamma^{2}}\right)\left(\gamma^{2} I-\alpha\left(\alpha L^{*} L+I\right)^{-1}\right)\right)$, and $\alpha, \gamma$ and $\gamma_{1}$ are real positive scalars such that $\gamma^{2} \geq$ $\frac{1}{\frac{1}{\alpha}+\lambda_{\min }\left(L^{*} L\right)}$ and $\gamma>\gamma_{1}$.

It should be noted that we have several degrees of freedom in choosing the parameters $\left(\gamma_{1}, \gamma, \alpha, B\right)$ to tighten the bound in (14) as much as possible. How to do this is currently under investigation. However, a simple choice is to let $\gamma_{1} \rightarrow \gamma$. This has two benefits: it maximizes the second term in (14) and it sets $B=0$ so that we need not worry about which square root matrix for $B$ to choose. Finally, to maximize the first term, we need to take $\gamma$ as its smallest value, i.e., $\gamma^{2}=\frac{1}{\frac{1}{0}+\lambda_{\min }\left(L^{*} L\right)}$.

The above discussion provides that the lower bound from (14) simplifies to

$$
\begin{aligned}
& L B_{\text {hin } f}=\left\|\Delta^{-1} \mathbf{b}\right\|^{2}-\frac{k-1}{4 \alpha}+ \\
& \min _{\mathbf{a} \in \mathcal{D}}\left(\frac{1}{\alpha}+\lambda_{\min }\left(L^{*} L\right)\right)\left\|\mathbf{a}-\alpha L^{*}\left(\alpha L L^{*}+I\right)^{-1} \mathbf{b}\right\|^{2} .
\end{aligned}
$$

Although we have already chosen parameters $\gamma_{1}$ and $\gamma$, the value of $L B_{\text {hinf }}$ in (15) is still dependent on the choice of the parameter $\alpha$. Ideally, $\alpha$ should be chosen so that it maximizes $L B_{\text {hinf }}$ in (15). However, this optimization appears to be computationally difficult, and hence we choose $\alpha$ as

$$
\hat{\alpha}=\arg \max _{\alpha}\left\|\Delta^{-1} \mathbf{b}\right\|^{2}-\frac{k-1}{4 \alpha} .
$$

With the choice of $\hat{\alpha}$ as in (16), we obtain the value of a lower bound for the integer least-squares problem (8) or (9) as

$$
L B_{\text {hinf }}= \begin{cases}L B_{\text {hinf }}^{(s p h)}+D L B & \text { if }\left\|\hat{\Delta}^{-1} \mathbf{b}\right\|^{2}-\frac{k-1}{4 \hat{\alpha}}>0 \\ 0 & \text { otherwise }\end{cases}
$$

where $L B_{\text {hinj }}^{(s p h)}=\left\|\hat{\Delta}^{-1} \mathrm{~b}\right\|^{2}-\frac{k-1}{4 \hat{\alpha}}, \hat{\Delta} \hat{\Delta}^{*}=\hat{\alpha} L L^{*}+I$, $\mathrm{b}=\mathbf{z}_{1: k-1}, L=R_{1: k-1,1: k-1}$, and $D L B=\min _{\mathrm{a} \in \mathcal{D} \subset Z^{k-1}}$ $\left(\frac{1}{\hat{\alpha}}+\lambda_{\min }\left(L^{*} L\right)\right)\left\|\mathbf{a}-\hat{\alpha} L^{*}\left(\hat{\alpha} L L^{*}+I\right)^{-1} \mathbf{b}\right\|^{2}$.
In the following section we will show that $L B_{h i n f}^{(s p h)}=$ ||$\hat{\Delta}^{-1} \mathbf{b}||^{2}-\frac{k-1}{4 \hat{\alpha}}$ with $\hat{\alpha}$ as defined in (16) is precisely the value of the lower bound on the integer least-squares problem in (8) or (9) if $\left\|\tilde{\Delta}^{-1} b\right\|^{2}-\frac{k-1}{4 \dot{\alpha}}>0$, in the case when the search space is relaxed to the sphere. This will mean that computing $L B_{\text {hinf }}^{(s p h)}$ will be of quadratic complexity as shown in [7]. Furthermore, computing $D L B$ is also of quadratic complexity per each node. The reason is that the inversion of $\left(\hat{\alpha} L L^{*}+I\right)$ only requires inversion of its eigenvalues. This latter operation is linear per node since the SVD decomposition of $L$, required for finding $L B_{\text {hinf }}^{(s p h)}$, can be done off-line as we discuss in the following section.

For the completeness we now state the modification of the sphere-decoding algorithm using lower bounds found based on the $H^{\infty}$ approach suggested in this section.

Input: $Q, R, x, y=Q^{*} x, d$.

1. Set $k=m, d_{m}^{2}=d^{2}, y_{m \mid m+1}=y_{m}$

2. (Bounds for $\left.s_{k}\right) \operatorname{Set} U B\left(s_{k}\right)=\left\lfloor\frac{\sqrt{d_{k}^{2}-L B_{h i n f, k}}+y_{k \mid k+1}}{r_{k, k}}\right\rfloor$, $s_{k}=\left\lceil\frac{-\sqrt{d_{k}^{2}-L B_{\text {hin } f, k}}+y_{k \mid k+1}}{\tau_{k, k}}\right\rceil-1$, where $L B_{\text {hinf }, k}$ is given by (17) (in (17), index $k$ which indicates the dimension of the problem is omitted for convenience; furthermore $L B_{h i n f, 1}=0$ )

3. (Increase $s_{k}$ ) $s_{k}=s_{k}+1$. If $s_{k} \leq U B\left(s_{k}\right)$ go to 5 , else go to 4.

4. (Increase $k$ ) $k=k+1$; if $k=m+1$ terminate algorithm, else go to 3 .

5. (Decrease $k$ ) If $k=1$ go to 6 . Else $k=k-1$, $y_{k \mid k+1}=y_{k}-\sum_{j=k+1}^{m} r_{k, j} s_{j}, d_{k}^{2}=d_{k+1}^{2}-\left(y_{k+1 \mid k+2}-\right.$ $\left.r_{k+1, k+1} s_{k+1}\right)^{2}-L B_{h i n f}$, and go to 2 .

6. Solution found. Save $s$ and its distance from $x, d_{m}^{2}-$ $d_{1}^{2}+\left(y_{1}-r_{1,1} s_{1}\right)^{2}$, and go to 3 .

\section{SPECIAL CASES}

In this section, we show how the lower bound derived in the previous section is related to the lower bounds based on the relaxation of the search space studied in [7].

\subsection{Spherical Relaxation}

Let us recall from [7] how the lower bound on the problem (8) can be evaluated in case when the search space is relaxed to a sphere. Let $L B_{s p h}=\left\|\mathbf{z}_{1: k-1}-R_{1: k-1,1 ; k-1} \hat{\mathbf{s}}_{1: k-1}\right\|_{2}^{2}$ where $\hat{s}_{1: k-1}$ is the solution of the following optimization 
problem

$$
\begin{aligned}
\min _{\mathbf{s}_{1: k-1}} & \left\|\mathbf{z}_{1: k-1}-R_{1: k-1,1: k-1} \mathbf{s}_{1: k-1}\right\|_{2}^{2} \\
\text { subject to } & \sum_{i=1}^{k-1} \mathrm{~s}_{i}^{2} \leq \frac{k-1}{4},
\end{aligned}
$$

Let SVD of $R_{1: k-1,1: k-1}$ be $R_{1: k-1,1: k-1}=U \Sigma V^{T}$ and let $\mathrm{q}=U \mathbf{z}_{1: k-1,1: k-1}$. Then if $\sum_{i=1}^{r}\left(\frac{\mathbf{q}_{i}}{\sigma_{i}}\right)^{2}>\frac{k-1}{4}$, the solution $\hat{\mathbf{s}}_{1: k-1}$ can be calculated as

$$
\hat{\mathbf{s}}_{1: k-1}=\sum_{i=1}^{r}\left(\frac{\sigma_{i} \mathbf{q}_{i}}{\sigma_{i}^{2}+\overline{\lambda^{*}}}\right) \mathbf{v}_{i}
$$

where $\lambda^{*}$ is obtained as a solution of

$$
\sum_{i=1}^{r}\left(\frac{\sigma_{i} \mathrm{q}_{i}}{\sigma_{i}^{2}+\lambda^{*}}\right)^{2}=\frac{k-1}{4}
$$

Clearly, if $\sum_{i=1}^{r}\left(\frac{g_{i}}{\sigma_{i}}\right)^{2} \leq \frac{k-1}{4}$, we obtain $L B_{s p h}=0$.

Now, it is not difficult to show that, upon differentiating (16), one obtains $1 / \hat{\alpha}=\lambda^{*}$ in the case when $\sum_{i=1}^{r}\left(\frac{g_{i}}{\sigma_{i}}\right)^{2}>$ $\frac{k-1}{4}$, which corresponds to $\left\|\hat{\Delta}^{-1} \mathbf{b}\right\|^{2}-\frac{k-1}{4 \hat{\alpha}}>0$. Furthermore, it is easy to show that in this case $L B_{s p h}=$ $L B_{\text {hinf }}^{(s p h)}=\left.|| \hat{\Delta}^{-1} \mathbf{b}\right|_{1} ^{2}-\frac{k-1}{4 \hat{\alpha}}$. When $\sum_{i=1}^{r}\left(\frac{q_{i}}{\sigma_{i}}\right)^{2} \leq \frac{k-1}{4}$, we obtain that $L B_{\text {sph }}=0$, and thus in the corresponding case $\left\|\hat{\Delta}^{-1} \mathbf{b}\right\|^{2}-\frac{k-1}{4 \hat{\alpha}} \leq 0$ in (17) we also have $L B_{\text {hinf }}=$ 0 . Since for $\left\|\hat{\Delta}^{-1} b\right\|^{2}-\frac{k-1}{4 \hat{\alpha}}>0$ from (17) follows that $D L B \geq 0$, we conclude that the lower bound $L B_{\text {hinf }}$ on the integer least-squares problem defined in (8) is tighter than the lower bound $L B_{s p h}$ on the same problem obtained with the relaxation of the search space to a sphere.

We may remark that had we started with the following $H^{\infty}$ problem

$$
\frac{\left\|\mathbf{b}-L K_{2} \mathbf{b}\right\|^{2}}{\frac{1}{\alpha}\|\mathbf{a}\|^{2}+\|\mathbf{b}-L \mathbf{a}\|^{2}} \leq \gamma^{2},
$$

then we would have immediately obtained the spherical relaxation. (10) is therefore more general in the sense that there is an extra term in the numerator.

To summarize, the lower bound (17) on the problem (8) is tighter than the one obtained relaxing the search space to a sphere. We formally refer to the use of the lower bound $L B_{\text {hinf }}$ defined in (17) in the sphere decoding algorithm as HINFSD-algorithm and to the use of the lower bound $L B_{s p h}$ as SPHSD-algorithm. Their performances are compared in Figure 1. The simulation results presented in Figure 1 are obtained with the radius initially chosen as suggested in [3] and updated each time we reach the bottom of the tree. Additionally, Schnor-Euchner strategy was employed in searching the tree points at each level. For the plots we present we have chosen $m=45$ and so snr $=$
$10 \log _{10} \frac{m}{4 \sigma^{2}}$, where $\sigma^{2}$ is the variance of each component of the noise vector $w$. As can be seen, the total flop count of the standard sphere decoder to which we refer as the SDalgorithm is improved at low SNRs. Also on Figure 1, the distributions of the number of visited nodes per level in the search tree are shown. As it can be seen the newly suggested algorithms prune the number of points in the tree by several order of magnitude. However, only HINFSD-algorithm improves the total flop count since it uses the tighter lower bound that the SPHSD-algorithm.
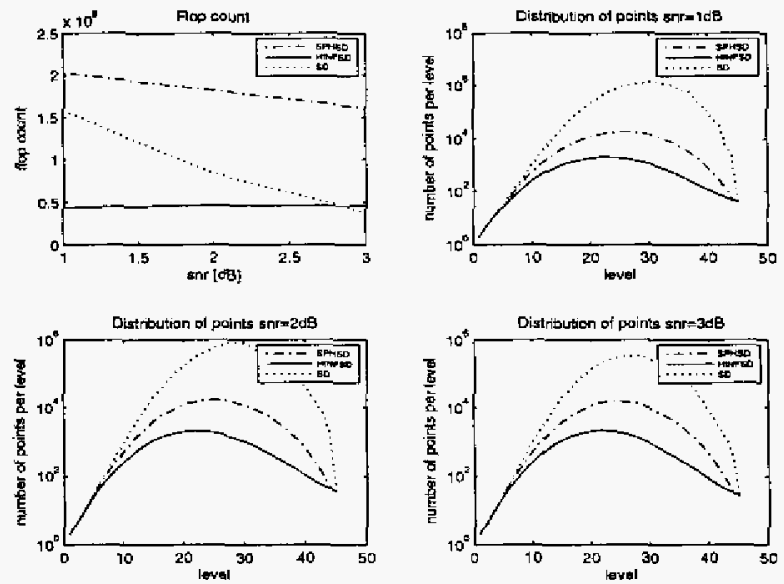

Fig. 1. H-infinity and sphere relaxation based lower bound for $m=45$

\subsection{Polytope relaxation}

In this subsection, we show that the lower bound on the integer least-squares problem (8) in case when the search space is relaxed to a polytope is yet another special case of the lower bound derived in Section 2.

Now, if we instead of (19) use

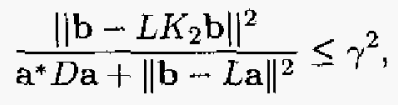

where $D$ is diagonal positive semi-definite matrix but with possibly different elements on the diagonal, we will clearly tighten the bound obtained using spherical relaxation. Furthermore, it is not too difficult to show that the bound defined in (20) cotresponds to the bound obtained by relaxing the search space to a polytope. The performance of the polytope relaxation, as well as the way to evaluate the lower bound in this case, is already analyzed in earlier work. We omit the details and refer the interested reader to [7].

\section{SUMMARY AND DISCUSSION}

In this paper, we attempted to improve the computational complexity of sphere decoding in the regimes of low SNR 
and/or high dimensions, by further pruning points from the search tree. The main idea is based on computing a lower bound on the remainder of the cost function as we descend down the search tree (the standard sphere decoder simply uses a lower bound of zero). If the sum of the current cost at a given node and the lower bound on the remaining cost from that node exceeds the cost of an already found solution, then that node (and all its descendants) are pruned from the search tree. In this sense, we are essentially using a "branch and bound" technique.

The main contribution of this paper is a new method of computing the lower bounds. The method we proposed is based on $H^{\infty}$ estimation. In general, the bounds based on this approach depend on the simultaneous choice of several parameters. One possible choice of these parameters results in a bound that is guaranteed to be better than the one obtained by simply relaxing the search space to a sphere. These lower bounds appear to be promising since, in an earlier work, we have noted that lower bounds tighter than the ones obtained by relaxing the search space to a sphere yet still being of quadratic computational complexity would improve the performance of the sphere decoder algorithm. We presented simulation results which demonstrated that the new lower bound, leads to a modified sphere decoding algorithm significantly faster than the standard sphere decoder algorithm in the regimes of low SNR and/or high dimensions.

Although we found a possible choice of the free parameters such that the new lower bound is better than the previously used spherical one, we did not address finding the best value of these parameters. This means that, in general, with a better choice of the parameters in (14), our lower bound may perform even better. Finding a better way of selecting these parameters is currently under investigation.

\section{REFERENCES}

[1] U. Fincke and M. Pohst, "Improved methods for calculating vectors of short length in a lattice, including a complexity analysis," Mathematics of Computation, vol. 44, pp. 463-471, April 1985.

[2] G. Golub and C. Van Loan, "Matrix Computations," John Hopkins University Press, 3rd edition, 1996.

[3] B. Hassibi, and H. Vikalo, "On the sphere decoding algorithm: part $\mathbf{I}$, the expected complexity," to appear in IEEE Trans. on Signal Processing.

[4] H. Artes, D. Seethaler and F. Hlawatsch, "Efficient detection algorithms for MLMO channels: A geometrical approach to approximate ML detection," IEEE Trans. on Signal Processing, November, 2003.
[5] R. Gowaikar and B. Hassibi, "Efficient maximumlikelihood decoding via statistical pruning", submitted to IEEE Trans. on Information Theory.

[6] M. O. Damen, A. Chkeif and J.-C. Belfore, "Lattice code decoder for space-time codes"' IEEE Comm. Let. pp. 161-163, May 2000.

[7] M. Stojnic, H. Vikalo and B. Hassibi, "A branch and bound approach to speed up the sphere decoder", ICASSP, Intemational conference on Acoustics, Speech and Signal Processing, Philadelphia, March, 2005.

[8] B. Hassibi, A. H. Sayed, and T. Kailath, IndefiniteQuadratic Estimation and Control, SIAM, 1999. 